CHLAMYDIA

\title{
Condom effectiveness for prevention of Chlamydia trachomatis infection
}

\author{
L M Niccolai, A Rowhani-Rahbar, H Jenkins, S Green, D W Dunne
}

Sex Transm Infect 2005;81:323-325. doi: 10.1136/sti.2004.012799

\begin{abstract}
Background/objectives: A growing body of evidence is increasingly demonstrating the effectiveness of condoms for sexually transmitted infection (STI) prevention. The purpose of the present analysis was to provide a disease specific estimate for the effectiveness of condoms in preventing Chlamydia trachomatis infection while controlling for known exposure to infection.

Methods: Condom effectiveness for $C$ trachomatis was estimated using a medical record database from a public sexually transmitted disease clinic $(n=1455)$. Clients were classified as having known exposure to $C$ trachomatis if they presented to the clinic as a contact to an infected partner. Results: Among clients with known exposure, 13.3\% of consistent condom users were diagnosed with $C$ trachomatis infection compared to $34.4 \%$ of inconsistent condom users (adjusted odds ratio $=0.10 ; 95 \% \mathrm{Cl}$ : 0.01 to 0.83 ). Among clients with unknown exposure, there was no observed protective effect of condoms.

Conclusions: This study provides further evidence that condoms are effective in preventing $C$ trachomatis infection by reporting a disease specific estimate and restricting analyses to individuals with known exposure.
\end{abstract}

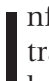
nfection with Chlamydia trachomatis is a common sexually transmitted infection (STI) that is associated with severe health consequences including pelvic inflammatory disease and infertility; ${ }^{1}$ therefore, its prevention remains an important public health priority. Though male condoms have proved efficacy for STI prevention in laboratory conditions, their effectiveness has been difficult to demonstrate in epidemiological studies. Limited recall and social desirability bias contribute to misreporting of condom use. Additionally, people may use condoms based on their perception of partner risk- that is, they tend to use condoms with partners that seem risky, resulting in confounding. ${ }^{2}$ Furthermore, studies are often limited by difficulties in assessing temporal relationships. ${ }^{3}$ A 2001 review of the scientific literature determined that there was insufficient evidence to draw definitive conclusions about condom effectiveness for the prevention of $C$ trachomatis. ${ }^{4}$

A more recent review by Holmes and colleagues highlights research since 2001 that supports condom effectiveness for $C$ trachomatis and other infections. ${ }^{3}$ Statistically significant reductions in infections among condom users have been observed among STD clinic populations (58\% reduction), ${ }^{5}$ population based samples (50\% reduction), ${ }^{6}$ young women (46\% reduction), ${ }^{7}$ and female sex workers $(26 \%$ reduction).$^{8}$ However, these studies are limited by either their lack of disease specific estimates ( $C$ trachomatis is often reported with other STI such as Neisseria gonorrhoeae and Trichomonas vaginalis), ${ }^{5-7}$ inability to assess the infection status of sex partners and therefore exposure in the sample population, ${ }^{6-8}$ or both. $^{67}$ Disease specific estimates are important because the strongest evidence for condom effectiveness can be attained when multiple outcomes (for example, different STI with distinct transmission and epidemiologic properties) are not combined. Furthermore, including an assessment of the exposure status of study participants (for example, sex with an infected partner) is critical because an individual who is not exposed to an STI cannot be infected whether condoms are used or not; therefore, these unexposed individuals do not contribute meaningful observations to the analysis. The purpose of this study was to provide an estimate of condom effectiveness for a single infection, $C$ trachomatis, among people who were exposed to infection.

\section{METHODS}

Beginning in the year 2000, the Connecticut Department of Public Health STD Control Program began collecting health information about clients on a standardised four page medical record form. This form collects information about demographics, reason for visit, risk behaviours, STI history, symptoms, signs, diagnoses, treatment, and counselling. These analyses included all initial client visits to the New Haven clinic during 2000-2. All participating institutional review boards approved this study.

The outcome of interest, $C$ trachomatis infection, was determined using DNA detection (Gen-Probe PACE 2, GenProbe, Inc, San Diego CA, USA) before July 2001 and DNA amplification (BD ProbeTec EC, Becton-Dickinson, Sparks MD) after July 2001. Condom use, the predictor of interest, was collected on the form as "always, sometimes, or never"; those who reported "always" using condoms were classified as consistent users. Exposure to an infected sex partner was determined by reason for visit as marked on the medical record form. Individuals who were designated "contact to chlamydia" were classified as having known exposure. Covariates of interest included age, sex, sexual orientation, race/ ethnicity, marital status, number of sexual partners in the previous 3 months, STI history, and symptoms of infection including discharge or dysuria. Continuous co-variates were dichotomised for ease of interpretation using established cutoff points to reflect the risk of infection associated with younger age and multiple partners.

The proportions of consistent and inconsistent condom users who were diagnosed with $C$ trachomatis were computed for those with known and unknown $C$ trachomatis exposure separately, and Pearson's $\chi^{2} \mathrm{p}$ values were computed. Logistic regression modelling was used to estimate unadjusted and adjusted odds ratios with 95\% confidence intervals (CI) for the association between condom use and $C$ trachomatis infection for known and unknown $C$ trachomatis exposure status separately. 


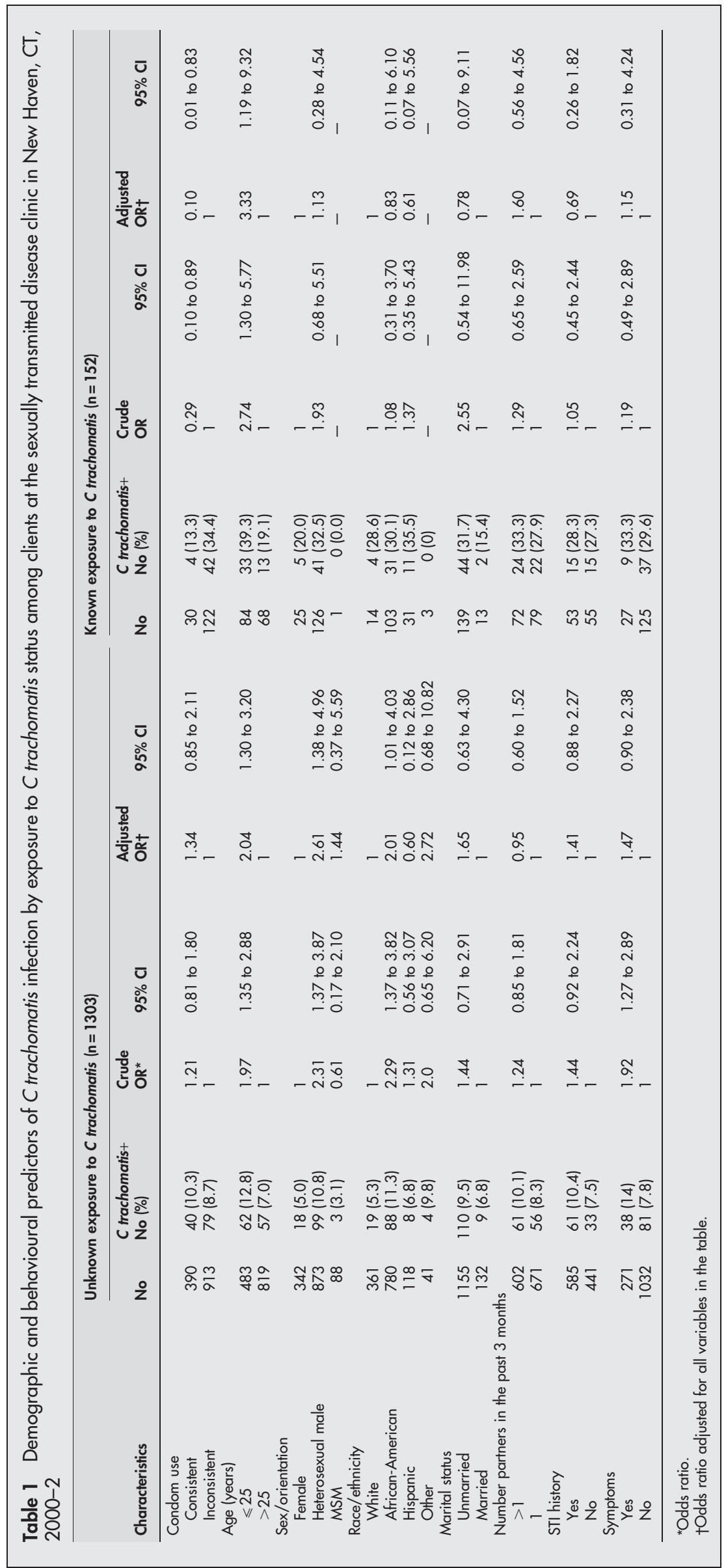




\section{RESULTS}

A total of 1870 initial visits were recorded during the study period. We excluded $415(22 \%)$ clients who had missing data; the majority $(\mathrm{n}=392,94 \%)$ were not tested for $C$ trachomatis based on clinician assessment of need, therefore constituting an appropriate exclusion. These individuals were more likely to be older, white, have fewer sex partners and no STI history. Therefore, the study population consisted of 1455 clients. The mean age of clients was 30.4 (SD 9.9) years. The majority of clients were male, African-American, and unmarried. Approximately $30 \%$ reported consistent condom use, and $11 \%$ were contacts to $C$ trachomatis. $C$ trachomatis was diagnosed in $165(11 \%)$ clients.

Among clients with known $C$ trachomatis exposure $(\mathrm{n}=152), 46(30 \%)$ were diagnosed with $C$ trachomatis infection, including $13.3 \%$ of consistent condom users and $34.4 \%$ of inconsistent condom users $(\mathrm{p}=0.02)$. Among clients with unknown $C$ trachomatis exposure $(n=1303)$, $119(7 \%)$ were diagnosed with $C$ trachomatis infection, including $10.3 \%$ of consistent condom users and $8.7 \%$ of inconsistent condom users $(\mathrm{p}=0.36)$. In the multivariate analysis including all covariates (table 1), consistent condom use was significantly associated with a $90 \%$ reduction in the prevalence of $C$ trachomatis infection among clients with known exposure (adjusted odds ratio $=0.10 ; 95 \%$ CI: 0.01 to 0.83 ). The effect among those with unknown exposure was not significant.

\section{DISCUSSION}

The results of this study indicate that consistent condom use is significantly associated with a $90 \%$ reduction in the prevalence of $C$ trachomatis infection among those with known exposure to an infected partner. The present analyses provide a more refined estimate than previously reported by restricting the analyses to a single infection, $C$ trachomatis, and by restricting the sample population to only those who reported exposure to infection. Indeed, our estimate exceeds others (90\% effective $v 26 \%$ to $58 \%$ effective), perhaps because our estimate was undiluted by the inclusion of people who do not contribute meaningful observations to the analyses-namely, individuals who may not have been exposed to $C$ trachomatis; their condom use would not be expected to provide protection. While our estimate is greater than the one estimated by Warner and colleagues who also only included contacts to infected people, the substantial overlap of the $95 \%$ confidence intervals indicates they are indeed quite consistent.

The use of an electronic medical record database was an efficient use of existing resources that allowed us to include all clients seen at this clinic from 2000-2, thereby reducing potential selection bias. However, the use of this data source also has some limitations. Firstly, our broad measure of condom use was based on self report and did not determine correct use or refer to frequency of sexual intercourse or a specific time period. Secondly, the cross sectional nature of this study limited our analyses to prevalent infections and did not allow us to determine the timing of sexual intercourse in relation to timing of infection in the partner. Thirdly, we were not able to distinguish the directionality of transmission between the clients and their partners. Finally, our findings may not be generalisable to non-STD clinic patients.

While the effective potential of condoms in preventing STIs is intuitive, the limitations of epidemiological research have made this difficult to demonstrate. The results of this study provide an improved estimate of condom effectiveness for prevention of $C$ trachomatis compared to previous reports that did not restrict analyses to the population at risk.

\section{CONTRIBUTORS}

LMN conceptualised the study, conducted data management, supervised the statistical analyses, and wrote the manuscript; AR-R assisted with concepts and data management, and conducted statistical analyses, and edited the manuscript; HJ developed the plan for and supervised the data collection and entry, and edited the manuscript; DWD and SG collected the data and edited the manuscript.

\section{Authors' affiliations}

L M Niccolai, A Rowhani-Rahbar, Yale University School of Medicine, Department of Epidemiology and Public Health, New Haven, CT, USA L M Niccolai, Yale University Center for Interdisciplinary Research on AIDS, New Haven, CT, USA

H Jenkins, Connecticut Department of Public Health, STD Control

Program, Hartford, CT, USA

D W Dunne, S Green, New Haven Health Department STD Clinic, New Haven, CT, USA

Competing interests: none declared.

The Human Investigations Committees at Yale University School of Medicine (New Haven, CT, USA), and Connecticut Department of Public Health (Hartford, CT, USA) approved access to and analyses of these data.

Correspondence to: Linda M Niccolai, PhD, Yale University Department of Epidemiology and Public Health, 60 College Street, PO Box 208034 New Haven, CT 06520-8034, USA; linda.niccolai@yale.edu

Accepted for publication 1 November 2004

\section{REFERENCES}

1 Holmes KK, Sparling PF, Mardh P-A, et al, eds. Sexually transmitted diseases. New York: McGraw-Hill, 1999.

2 Peterman TA, Lin LS, Newman DR, et al. Does measured behavior reflect STD risk? An analysis of data from a randomized controlled behavioral intervention study. Sex Transm Dis 2000;27:446-51.

3 Holmes KK, Levine R, Weaver M. Effectiveness of condoms in preventing sexually transmitted infections. Bull World Health Organ 2004:82:454-61.

4 National Institute of Allergy and Infectious Diseases, National Institutes of Health, Department of Health and Human Services. Workshop summary: scientific evidence on condom effectiveness for sexually transmitted disease prevention. 20 July 2001.

5 Warner L, Newman DR, Austin HD, et al. Condom effectiveness for reducing transmission of gonorrhea and chlamydia: The importance of assessing partner infection status. Am J Epidemiol 2004;159:242-51.

6 Ahmed S, Lutalo T, Wawer M, et al. HIV incidence and sexually transmitted disease prevalence associated with condom use: a population study in Rakai, Uganda. AIDS 2001;15:2171-9.

7 Crosby RA, DiClemente RJ, Wingood GM, et al. Value of consistent condom use: a study of sexually transmitted disease prevention among African American adolescent females. Am J Public Health 2003;93:901-2.

8 Sanchez J, Campos PE, Courtois B, et al. Prevention of sexually transmitted diseases (STDs) in female sex workers: prospective evaluation of condom promotion and strengthened STD services. Sex Transm Dis 2003;30:273-9. 